\title{
PROGRAMA SEIS SIGMA: ASPECTOS SINÉRGICOS COM OUTRAS ABORDAGENS DE GERENCIAMENTO DA QUALIDADE
}

\section{SIX SIGMA PROGRAM: SYNERGIC ASPECTS WITH OTHERS QUALITY MANAGEMENT APPROACHES}

\section{Silvia Helena Boarin Pinto}

Pós-Doutoranda do Depto. de Engenharia de Produção - Escola Politécnica da USP Av. Prof. Almeida Prado, 128 - CEP 05508-900 São Paulo, SP Brasil Telefone (011) 30915363 Fax 30915399 e-mail: silvia.boarin@pesquisador.cnpq.br

\section{Marly Monteiro de Carvalho}

Professoras Doutoras do Depto. de Engenharia de Produção - Escola Politécnica da USP Av. Prof. Almeida Prado, 128 - CEP - 05508-900 São Paulo, SP Brasil Telefone (011) 30915363 R. 303 Fax 30915399 e-mail: marlymc@usp.br

\section{Linda Lee Hoo}

Professoras Doutoras do Depto. de Engenharia de Produção - Escola Politécnica da USP Av. Prof. Almeida Prado, 128 - CEP - 05508-900 São Paulo, SP Brasil Telefone (011) 30915363 R. 303 Fax 30915399 e-mail: lindalee@usp.br 


\title{
RESUMO
}

Diversas abordagens de gestão da qualidade têm sido adotadas em todos os continentes há décadas. Uma das mais recentes, o programa Seis Sigma, vem sendo implementado por um número crescente de empresas. O Seis Sigma surge no atual cenário, em que as organizações realizam grandes investimentos na implementação de programas de melhoria da qualidade e se deparam com situações críticas como a mensuração do retorno sobre o capital investido, bem como a análise criteriosa dos benefícios alcançados. A proposta deste artigo é identificar as principais características do Seis Sigma, discutir quais os fatores críticos na sua implementação, as ferramentas e técnicas que suportam este processo, bem como sua relação com outros consagrados programas de melhoria da qualidade. A abordagem metodológica utilizada foi o estudo de múltiplos casos, desenvolvido em dez grandes empresas brasileiras de diferentes setores industriais. A pesquisa concluiu que existe sinergia entre o Seis Sigma e os demais programas e as organizações que adotaram o Seis Sigma obtiveram benefícios financeiros e de qualidade.

Palavras-chave: Programas de Melhoria da Qualidade, Seis Sigma.

\begin{abstract}
Several approaches in quality management have been adopted worldwide for decades. One of the latest approaches, Six Sigma quality program, has been increasingly implemented by several companies. Six Sigma emerges today in a scenario in which enterprises have been investing heavily in the implementation of quality programs, but have to cope with critical situations such as the measurement of returns on invested capital as well as the extensive analysis of the accomplished benefits. The aim of this article is to identify Six Sigma main features, to determine the critical factors in its implementation, the tools and techniques supporting this process, and also its relationship with other well-known quality programs. The methodological approach used was the study of different cases in ten large companies from different industrial sectors. The study concluded that there is synergy between Six Sigma and the other programs and that the companies in which Six Sigma was implemented achieved both financial and quality benefits.
\end{abstract} Key-words: Quality Programs, Six Sigma. 


\section{INTRODUÇÃO}

O atual ambiente organizacional está sofrendo transformações cada vez mais rápidas, necessitando, portanto, de estratégias competitivas e de gestão com contínuo aprimoramento para as organizações poderem concorrer e sobreviver. É necessário oferecer produtos e serviços diferenciados, melhorar a eficiência e gerar e manter vantagens competitivas, através de estratégias de manufatura que devem estar condizentes com as estratégias do negócio. As estratégias de manufatura colaboram para melhorar a competitividade das empresas, utilizando para isso a redução de custos, a melhoria contínua da qualidade e da produtividade de produtos e serviços, dentre outros aspectos, criando uma vantagem competitiva sustentável, pois em função de suas características específicas e complexas podem oferecer uma distinção em relação à concorrência, conforme Prahalad e Hamel (1990).

Um dos principais componentes da estratégia da manufatura é a qualidade que sofreu diversas alterações em sua formulação e no final do século XX e início do século XXI. Os motivos que acarretaram tais mudanças foram: a acirrada competição entre as empresas de todo o mundo; o surgimento dos grandes blocos econômicos; e a desaceleração do crescimento econômico mundial, em face da consolidação da qualidade de produtos e serviços como sendo fundamental para a sobrevivência das empresas.

A implantação de normas de gestão da qualidade como a ISO 9001:2000; a norma de gestão ambiental ISO 14001; dos prêmios de qualidade como o Malcolm Baldrige (americano), o PNQ - Prêmio Nacional de Qualidade (brasileiro), o EFQM - Prêmio Europeu da Qualidade; de programas de qualidade como o Seis Sigma e o TQM - Total Quality Management constituem uma alternativa bastante viável para as empresas poderem concorrer nos moldes do mercado atual.

Diversos autores como Reed et al.(2000), Hendricks e Singhall (1997) e Hendricks e Singhall (2001), indicam que a adoção efetiva de programas de melhoria da qualidade pode gerar resultados positivos, criando vantagens competitivas sustentáveis para as empresas. Contudo, estudos demonstram a existência de diversas dificuldades na correta implementação desses programas (Kaynak, 2003). 


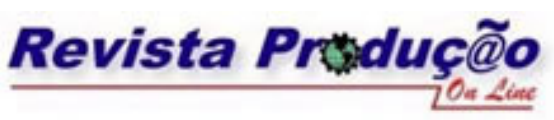

Associação Brasileira de Engenharia de Produção - ABEPRO

Universidade Federal de Santa Catarina - UFSC

www.producaoonline.org.br

ISSN 1676 - 1901 / Vol. IX/ Num.I/ 2009

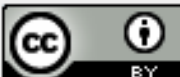

Estudos empíricos conduzidos por Hendricks e Singhall (1997) indicaram que a efetiva adoção dos programas de melhoria da qualidade pode trazer impacto positivo no crescimento das vendas e na redução dos custos. Quatro anos mais tarde os mesmos autores realizaram outra pesquisa, dessa vez utilizando como parâmetro de análise o comportamento das ações dessas empresas, verificando que o desempenho das mesmas teve uma performance superior (Hendricks e Singhall, 2001).

Pesquisas empíricas em empresas localizadas no Brasil ainda são raras, ou seja, são necessárias avaliações para um melhor entendimento da realidade brasileira sobre o tema. Portanto, neste artigo é apresentado um estudo de múltiplos casos em 10 grandes organizações no Brasil, tendo como objetivo extrair evidências empíricas de que os investimentos aplicados em qualidade trazem retornos financeiros, além de identificar as principais tendências em curso nos programas de melhoria da qualidade e os principais gargalos no processo de implementação dos mesmos.

O artigo está estruturado em quatro seções. As duas primeiras apresentam a síntese da discussão teórica dos temas abordados neste trabalho e a abordagem metodológica proposta para a pesquisa de campo, respectivamente. A seção 3 demonstra os resultados do levantamento verificado no estudo de múltiplos casos. Finalmente, a seção 4 traz as conclusões, as limitações e as recomendações para trabalhos futuros.

\subsection{Os programas de melhoria da qualidade}

Os modelos de gestão da qualidade evoluíram e se difundiram ao longo do último século. Os teóricos da área de qualidade tiveram muita influência nas organizações e os principais pensadores são: Juran, Feingenbaum, Deming, Crosby, Ishikawa, dentre outros. A abordagem de qualidade nas organizações passou por várias eras desde um enfoque de inspeção, passando pelo controle, garantia e gestão da qualidade (Garvin, 1988).

Neste trabalho, foram analisados três modelos de gestão de qualidade, as normas ISO 9001:2000, o Total Quality Management (TQM) e o Seis Sigma.

As normas internacionais da série ISO 9000 representam um conjunto de diretrizes mundialmente conhecidas e implantadas que estabelecem a gestão e a garantia da qualidade, podendo ser aplicadas a produtos e serviços, independentemente do porte ou setor da 
organização. Desde sua criação, na década de oitenta, as referidas normas produziram um grande impacto mundial no comércio internacional e também na implementação e manutenção de sistemas da qualidade nas organizações em todo o mundo, tornando-se uma referência global. As normas abordam os Sistemas de Gestão utilizados pelas organizações, incluindo o projeto, a produção, o fornecimento de produtos e os serviços associados. A metodologia PDCA, inicialmente desenvolvida por Shewhart em 1920 (Plan, Do, Check and Act, que em português representa planejamento, execução, verificação e ação de uma determinada atividade ou tarefa, podendo ser aplicada para produtos ou serviços), foi inserida como meio facilitador do entendimento da norma, possibilitando assim uma melhor disseminação da mesma.

A adoção das normas ISO 9000 já foi alvo de criticas no que concerne a relação custobenefício, por necessitar de investimentos significativos para sua implantação e, muitas vezes, não possuir retorno condizente. Segundo diversos autores, isso acontece principalmente nas organizações que adotaram as referidas normas apenas para cumprirem requisitos governamentais, como ferramenta de marketing ou por exigências de seus clientes.

Segundo Chow-Chua et al. (2003) e Rajan e Tamimi (2003), as empresas que realizaram a certificação ISO 9000 somente para participarem de determinadas concorrências ou para poderem vender seus produtos a entidades governamentais, consumiram altos recursos financeiros e humanos com o processo e o retorno nem sempre foi vantajoso.

Estudos demonstram que, nas empresas em que a adoção foi motivada pela busca contínua da excelência em qualidade e nas organizações que realizaram a certificação por outros motivos, após a devida adequação, o retorno financeiro e a melhoria da imagem da organização foram bastante significativos (Wilson et al., 2003).

A procura por vantagens competitivas para a sobrevivência das organizações no cenário atual encoraja as empresas a utilizarem processos e estruturas mais eficazes, adotando modelos de gestão que proporcionem melhorias na qualidade dos produtos e serviços, aliadas à redução significativas de custos. Dentre os modelos mais adotados mundialmente, destaca-se o TQM Total Quality Management. Sua origem foi no Japão, no final da Segunda Grande Guerra Mundial, oriundo da elevada competição japonesa na época, que buscava altos padrões de qualidade e produtividade a um custo comedido. O TQM utiliza práticas e técnicas como a diminuição dos retrabalhos, um novo planejamento dos processos organizacionais, o 
benchmarking, a análise contínua dos resultados e uma relação de proximidade com fornecedores. O TQM é uma abordagem que objetiva a melhoria constante da competitividade, da eficácia e da flexibilidade organizacionais. Compõe-se basicamente do planejamento, organização e compreensão de cada atividade a ser realizada, é dependente de cada colaborador em cada nível organizacional da empresa, e prega o respeito e a valorização do ser humano em todos os departamentos da empresa (Beheshti e Lollar, 2003; Montes et al., 2002; Power, 1997 e Projogo e Sohal, 2004).

Para o sucesso do TQM, deve-se trabalhar com sinergia em todos os setores da organização, visando sempre ao cumprimento das metas pré-estabelecidas e reconhecendo que cada funcionário e cada atividade afetam os outros e, por sua vez, são também afetados por eles, ou seja, a motivação e a valorização do ser humano é de total importância. Outro fator de extrema relevância é o treinamento contínuo dos funcionários (Guillén, 1994; Lee e Lazarus, 1996 e Spencer, 1996).

O Seis Sigma denota uma abordagem oriunda dos conceitos da Gestão da Qualidade Total - TQM, representando uma estratégia e uma metodologia de implantação de técnicas estatísticas (pensamento estatístico para redução da variabilidade dos processos) e métodos. Sua origem deuse na organização americana Motorola, que é proprietária da marca, nos anos 80 com o objetivo de auxiliar a resolver seus problemas de qualidade e lucratividade, pois a referida empresa já havia implantado diversos programas de qualidade e obtivera poucos resultados positivos. Em 1998, com a divulgação dos ganhos financeiros, acima de 1,3 bilhões de dólares, atribuídos ao programa Seis Sigma, a estratégia difundiu-se pelas empresas americanas, alcançando, posteriormente, o meio acadêmico e empresarial como um todo. A semelhança do TQM, o Seis Sigma é definido de diversas formas. Alguns autores sustentam que o Seis Sigma é a metodologia da qualidade para o século XXI. Comumente definido como uma estratégia gerencial muito disciplinada e quantitativa tendo como objetivo o aumento significativo da lucratividade das organizações, através da melhoria da qualidade, do aumento da satisfação dos clientes e dos consumidores (Blakeslee, 2001; Eckes, 2001; Hoerl e Snee, 2002; Pande et al., 2001, Pfeifer et al., 2004 e Snee, 2004).

O Seis Sigma representa uma implementação disciplinada, precisa e focalizada nos princípios e técnicas comprovadas da qualidade. Possui diversas ferramentas e sistemáticas de 


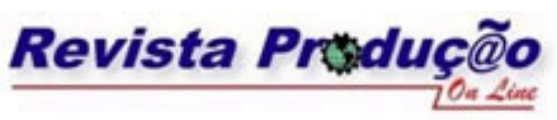

Associação Brasileira de Engenharia de Produção - ABEPRO

Universidade Federal de Santa Catarina - UFSC

www.producaoonline.org.br

ISSN 1676 - $\frac{\text { whw.prod. IX/ Num.I/ } 2009}{1901 \text { Vol. }}$

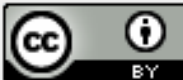

trabalho propostas por muitos estudiosos da qualidade, e objetiva o desempenho dos negócios livre de erros, sendo medido pelo desvio padrão de seus processos de negócios. O objetivo do Seis Sigma é o desempenho de 99,99966\% de conformidade, isto é, aproximadamente 3 nãoconformes /defeituosos por milhão de itens produzidos, mesmo havendo um deslocamento da média do processo em relação ao valor nominal em 1,5 desvios-padrão (sigma), classificando assim a empresa como uma organização de "classe mundial". Diversos autores enunciam que a maioria das empresas possui seus produtos e serviços operando entre 3 e 4 sigmas. Um dos fatores que estabelecem o sucesso dos programas Seis Sigma é a sua estrutura de implantação. Portanto, devem compreender a sua forma de condução: uma metodologia de solução de problemas e de desenvolvimento de novos produtos ou serviços (as cinco fases do DMAICdefine, measure, analyse, improve and control e o DFSS - Design for Six Sigma); uma estrutura de responsabilidade e funções para o programa; uma vigorosa estrutura de treinamentos; e uma política adequada de pessoal para sensibilizar os colaboradores para a mudança de cultura (Bassu, 2004, Carvalho e Paladini, 2005, Pande et al., 2001, Li-Hsing e Chen-Chia, 2006, Linderman et al., 2006 e Ravichandran, 2006).

\section{METODOLOGIA}

O presente trabalho utilizou-se, como estratégia de pesquisa qualitativa, uma abordagem de estudo de múltiplos casos, pois se julgou mais adequada às proposições e questões da pesquisa.

Observa-se que o estudo de múltiplos casos deve atender à lógica da replicação dos casos e não de amostragem. Yin (2004) argumenta que os estudos de caso e os experimentos podem ser generalizáveis em termos de proposições teóricas e não para populações ou universos.

Os principais critérios para a seleção das organizações que fizeram parte dos casos estudados foram: haver implementado a sistemática Seis Sigma há mais de dois anos, ou seja, o referido programa não está na fase de implantação; serem de distintos setores industriais e representarem empresas de capital origem nacional e multinacional. Com base nestes critérios foram selecionadas dez empresas das seguintes áreas: serviços médicos, siderurgia, equipamentos 


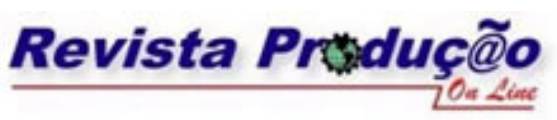

Associação Brasileira de Engenharia de Produção - ABEPRO

Universidade Federal de Santa Catarina - UFSC

www.producaoonline.org.br

ISSN 1676 - 1901 / Vol. IX/ Num.I/ 2009

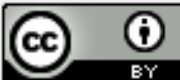

de transporte, energia, farmacêutica, telefonia, equipamentos diversos, químico, metalúrgico e de mineração, sendo quatro organizações de capital nacional e seis de origem multinacional.

O questionário adotado neste trabalho para a coleta de dados nas entrevistas realizadas nas organizações teve como principais fases: o levantamento bibliográfico das principais características dos programas de qualidade, visando ao conhecimento prévio para a elaboração das questões; a confecção preliminar das perguntas a serem inquiridas nas empresas, totalizando 30 questões; e a realização de um pré-teste, conforme a proposta de Cooper e Schindler (2003), visando excluir as perguntas com dupla interpretação, diversos tipos de erros, e também com o objetivo de melhorar a formulação das mesmas, dentre outros problemas.

O questionário, durante o pré-teste, foi aplicado em duas organizações não incluídas nas dez empresas do estudo de múltiplos casos, durante uma entrevista, o que possibilitou uma ampla verificação dos questionamentos realizados. A pesquisa foi realizada no segundo semestre do ano de 2005. As proposições centrais deste trabalho são:

$\left.\mathrm{P}_{1}\right) \mathrm{O}$ conjunto de ferramentas básicas da qualidade utilizado nas organizações pesquisadas para o Seis Sigma é o mesmo para os demais programas como a ISO 9001:2000.

$\mathrm{P}_{2}$ ) Existe sinergia entre o Seis Sigma e os demais programas de qualidade, quando utilizados simultaneamente nas empresas.

$\mathrm{P}_{3}$ ) A implantação do Seis Sigma acarretou benefícios financeiros significativos para as empresas que o adotou.

\subsection{Protocolo da pesquisa dos casos}

O protocolo de pesquisa adotado nas entrevistas realizadas nas 10 (dez) grandes empresas foi composto de:

- seleção das possíveis empresas a serem visitadas. Inicialmente, foram escolhidas 30 organizações, que atendessem aos critérios de seleção apresentados anteriormente. Posteriormente, foram realizados contatos telefônicos com o responsável pelo setor de qualidade das empresas selecionadas, visando apresentar os objetivos da pesquisa e solicitar uma visita para a condução da entrevista. Entre trinta empresas inicialmente escolhidas, 10 empresas aceitaram fazer parte da pesquisa; 
- realização das visitas e aplicação do questionário. As entrevistas foram conduzidas com os responsáveis pelos programas de qualidade, sendo executadas também verificações em diversos setores das empresas, como: produção, qualidade, recursos humanos, informática, dentre outros, visando constatar verificações in loco;

- registro das anotações complementares ao instrumento de pesquisa, com o objetivo de documentar as particularidades das organizações analisadas; e

- análise geral dos dados coletados, visando à validação das proposições estabelecidos.

\section{ANÁLISE DOS RESULTADOS}

O estudo de múltiplos casos foi caracterizado pela visita a dez empresas, onde foram realizadas as entrevistas. As organizações foram escolhidas por possuírem histórico de implantação de programas de qualidade, preferencialmente as normas ISO 9001:2000 e o Seis Sigma, implantados há pelo menos dois anos; um faturamento superior a 50 milhões de reais anuais; serem de diferentes ramos de atividade; com mais de 250 funcionários e haver formação de capital de origem nacional (N) e multinacional (M).

A Tabela 1 apresenta as principais características das dez empresas estudadas, compatíveis com os pré-requisitos da pesquisa. 
Tabela 1 - Características das Empresas

\begin{tabular}{|c|c|c|c|c|c|c|c|c|c|c|}
\hline \multirow{2}{*}{ 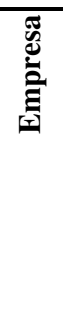 } & \multirow{2}{*}{ 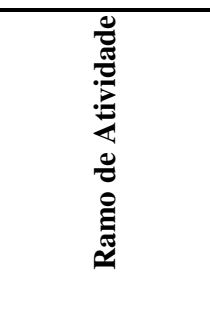 } & \multirow{2}{*}{ 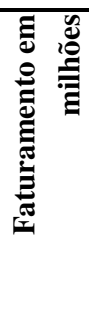 } & \multirow{2}{*}{ 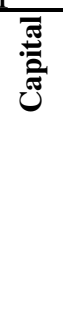 } & \multicolumn{7}{|c|}{ Programas de qualidade implantados } \\
\hline & & & & 양 & $\begin{array}{l} \\
0\end{array}$ & 영 & $\begin{array}{l}8 \\
\vdots \\
2 \\
2\end{array}$ & $\sum_{0}$ & 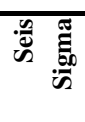 & $\stackrel{0}{0}$ \\
\hline 1 & Serviços médicos & $>50$ & $\overline{\mathrm{N}}$ & $\bar{x}$ & $\bar{x}$ & & & & $\bar{x}$ & $\mathrm{x}$ \\
\hline 2 & Siderúrgica & $>50$ & $\bar{N}$ & $\bar{X}$ & & $\bar{X}$ & & & $\bar{x}$ & \\
\hline 3 & $\begin{array}{l}\begin{array}{l}\text { Equipamentos de } \\
\text { transporte }\end{array} \\
\end{array}$ & $>50$ & $\bar{M}$ & $\bar{X}$ & & & & & $\bar{x}$ & \\
\hline 4 & Energia & $>50$ & $\mathrm{M}$ & $\mathrm{X}$ & & & & & $\mathrm{X}$ & \\
\hline 5 & Farmacêutica & $>50$ & $\bar{N}$ & $\bar{X}$ & $\bar{X}$ & & & & $\bar{x}$ & $\bar{X}$ \\
\hline 6 & Telefonia & $>50$ & $\bar{M}$ & $\bar{x}$ & & & & & $\bar{x}$ & \\
\hline 7 & $\begin{array}{l}\text { Equipamentos } \\
\text { diversos }\end{array}$ & $>50$ & $\bar{M}$ & $\bar{X}$ & $\bar{X}$ & & & $\bar{X}$ & $\bar{x}$ & \\
\hline 8 & Químico & $>50$ & $\bar{M}$ & $\bar{x}$ & $\bar{x}$ & & & $\bar{x}$ & $\bar{x}$ & $\bar{x}$ \\
\hline 9 & Metalúrgico & $>50$ & $\bar{N}$ & $\bar{X}$ & $\bar{X}$ & $\bar{X}$ & & & $\bar{X}$ & \\
\hline 10 & Mineração & $>50$ & $\bar{M}$ & $\bar{x}$ & $\bar{x}$ & & $\bar{x}$ & $\bar{x}$ & $\bar{x}$ & \\
\hline
\end{tabular}

Fonte: as autoras

Em todas as empresas analisadas, houve a certificação ISO 9001:2000 anterior à implantação da estratégia Seis Sigma. A justificativa apontada para essa situação pelos responsáveis por tais programas é que a norma ISO 9001:2000 fornece uma base para o Seis Sigma, ou seja, estabilizam os processos, estabelecem uma sistemática para elaboração e controle da documentação da empresa, proporciona uma certa experiência no trato com ações corretivas e preventivas, promovem uma cultura de melhoria contínua dos processos.

A Figura 1 apresenta a análise da utilização do Programas de qualidade estratificada segundo a origem do capital. Pode-se observar que apenas empresas multinacionais adotam a QS 9000 (1 empresa) e o TQM (3 empresas). Por outro lado, apenas as empresas nacionais adotam a ISO 16949 (2 empresas). 

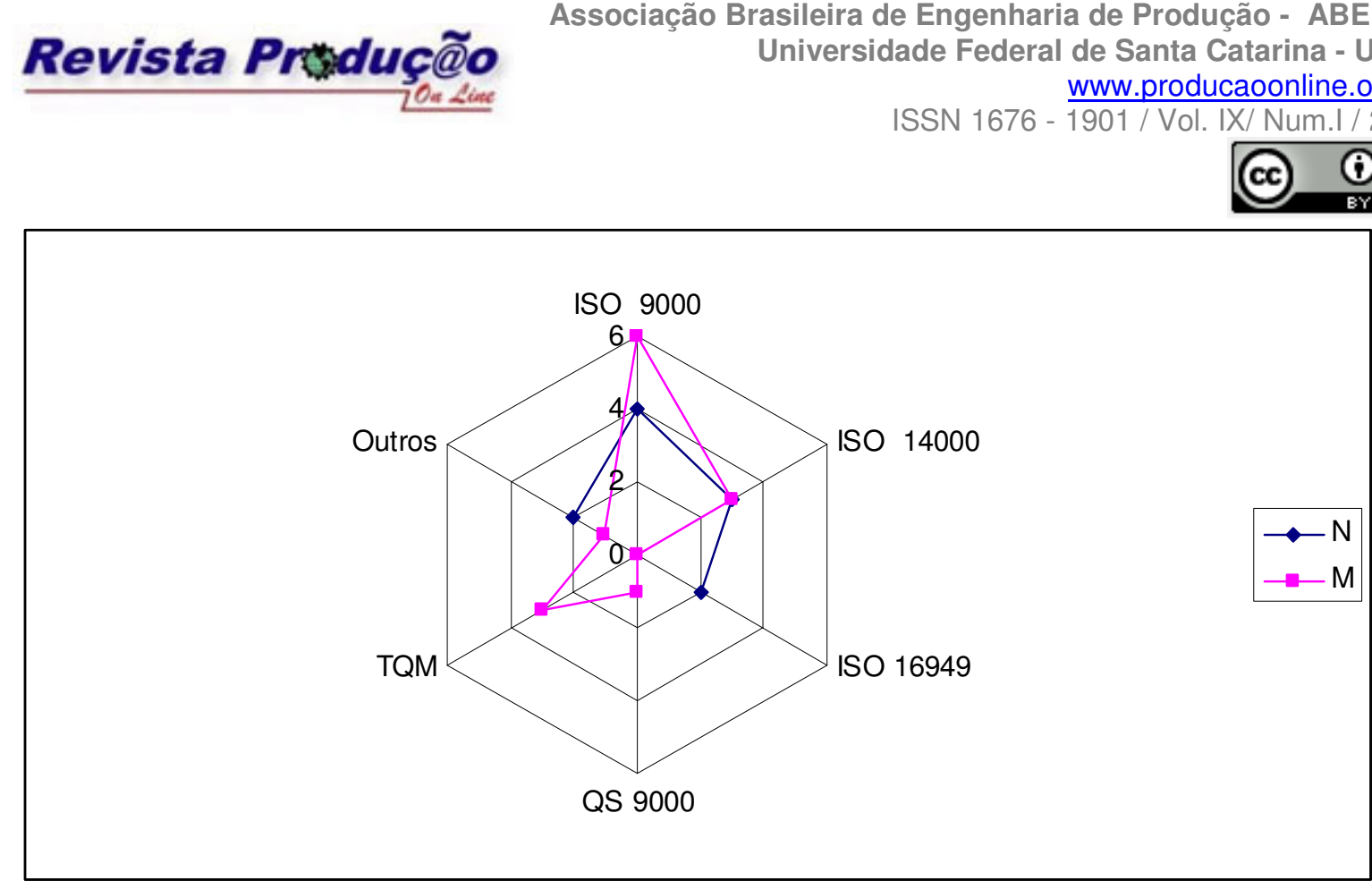

Figura 1 - Adoção de Programas de Qualidade segundo origem do capital Fonte: as autoras

Todos os coordenadores afirmaram que o Seis Sigma e as normas ISO 9001:2000 se complementam e devem coexistir concomitantemente nas organizações, acarretando a otimização dos recursos humanos e financeiros.

A seguir, serão listadas diversas características verificadas na implantação e manutenção do Seis Sigma nas organizações pesquisadas, onde foram realizadas entrevistas pessoais e verificações in loco.

O envolvimento da direção da empresa é identificado como sendo "muito alto". Uma explicação para tal aspecto pode ser o rápido e grande retorno financeiro ocasionado pelo mesmo, disponível na ampla literatura técnica sobre o tema e também em periódicos não técnicos.

A contratação de consultorias de grande porte foi verificada nas empresas nacionais, para apoiar a implantação, em parte do programa ou na sua totalidade. As consultorias realizaram diversos tipos de treinamentos e auxiliaram na implantação. Nas organizações multinacionais, foi utilizada a experiência de suas matrizes e também, em alguns casos, a contratação de consultorias no Brasil, pois essa última representou uma opção financeira mais acessível.

As principais dificuldades verificadas foram a elaboração e execução dos treinamentos internos; a complexidade das operações realizadas que demandam vastos estudos para os projetos Seis Sigma; a disponibilidade de tempo dos funcionários e a escolaridade dos colaboradores 
operacionais, principalmente nas empresas dos setores de aços para a construção, metalurgia e mineração, onde a utilização de mão de obra de baixa qualificação é verificada em diversos departamentos.

As principais facilidades verificadas foram a experiência do pessoal interno para a implantação e manutenção dos programas de qualidade; a disponibilidade de recursos financeiros para diversos fins; a escolaridade dos colaboradores que coordenam os programas; os pacotes de programas estatísticos adotados; a quantificação dos ganhos financeiros dos projetos; o histórico de implantação de outros programas de qualidade adotados anteriormente e as diversas ferramentas de qualidade.

Os motivos que impulsionaram a implantação do Seis Sigma foi a real possibilidade de substanciais ganhos financeiros, a curto e médio prazo, acrescidos dos benefícios na melhoria da qualidade e da produtividade.

Os treinamentos e a divulgação do programa em todos os casos analisados foram bastante apoiados pela direção das empresas e foi verificado que há um grande planejamento anual para a realização dos treinamentos internos, tanto na implantação do programa quanto para a manutenção do mesmo. Em certos casos (empresas dos setores de aços para a construção, metalurgia e mineração), tais treinamentos estão relacionados às promoções e benefícios salariais, ou seja, se os colaboradores não cumprirem as horas dos cursos estabelecidas para cada função, não poderão ser promovidos ou receberem aumentos salariais. Para os coordenadores dos programas nas empresas nacionais, optou-se pelo treinamento no Brasil com consultorias especializadas. Para os coordenadores dos programas, nas companhias multinacionais optou-se pelo treinamento em suas matrizes e, somente em um dos casos, o treinamento foi realizado no Brasil, com a utilização de consultorias técnicas renomadas, devido aos altos custos dos mesmos no exterior. A divulgação dos programas utilizou, em grande escala, palestras internas, comunicações impressas como cartas assinadas pelo presidente, workshops e, principalmente, cursos internos ministrados por funcionários devidamente qualificados ou pelas consultorias contratadas.

Em todos os casos envolvidos houve uma grande divulgação sobre o programa Seis Sigma e também uma carga horária de treinamentos substancialmente elevada, quando comparado aos demais programas implantados anteriormente (ISO 9001:2000, TQM, dentre 
outros). A explicação fornecida nas empresas foi a existência de uma maior disponibilidade de recursos financeiros e pessoais para diversas finalidades e o forte apoio da direção, ocasionando um impacto maior, quando comparado com os demais programas de qualidade adotados pela empresa anteriormente.

A receptividade ao programa foi classificada como "alta" e "muito alta". A explicação fornecida nas empresas pesquisadas foi a existência de uma maior e mais completa estratégia de divulgação do programa Seis Sigma (cartas do presidente da empresa explicando a metodologia e solicitando a participação dos colaboradores; cartilhas especialmente confeccionadas; palestras internas; workshops; inúmeros treinamentos; dentre outros), um amplo suporte financeiro e um grande apoio motivacional da direção.

A verificação das expectativas dos clientes em todas as empresas estudadas foi realizada através da "pesquisa de satisfação dos clientes" como um principal meio para a verificação das aspirações de seus consumidores. As pesquisas foram coordenadas por organizações externas, especializadas na área. Também foram avaliadas as respostas obtidas nos "serviços de atendimento ao cliente", estas, porém, em menor intensidade.

Com relação aos valores financeiros investidos e ganhos obtidos, todas as empresas foram unânimes em atestar que o referido programa realmente acarretou ganhos financeiros substanciais, já nos primeiros anos de sua implantação, sendo que o valor total investido foi extremamente inferior aos ganhos proporcionados com o referido programa.

A perspectiva de futuro do Seis Sigma nas organizações analisadas é de ampliação para setores pouco explorados até então, como por exemplo, nas áreas financeiras, administrativas e comerciais.

As principais alterações verificadas após a implantação do Seis Sigma foi o substancial ganho financeiro.

As interações entre os diversos programas de qualidade foram verificadas, principalmente entre a ISO 9001:2000 e o Seis Sigma. Os coordenadores dos programas enfatizaram os seguintes aspectos:

- o Seis Sigma representa uma ótima ferramenta de gestão para a melhoria contínua da qualidade (item que deve ser obrigatoriamente demonstrado na norma ISO 9001:2000); 
- diversas estruturas devem ser compartilhadas entre os programas como, por exemplo, a elaboração e controle de documentos, as sistemáticas de análise crítica, as ações corretivas e preventivas, dentre outras, diminuindo custos e otimizando recursos;

- as auditorias internas do sistema de qualidade da ISO 9001:2000 podem auxiliar na verificação da veracidade e na quantificação dos ganhos com os projetos Seis Sigma;

- os objetivos da qualidade estipulados para garantir que o planejamento do sistema de gestão esteja de acordo com as metas estabelecidas pela direção podem ser utilizados como os DPMO’s (Defeito por Milhão de Oportunidades) do Seis Sigma;

- a ISO 9001:2000 estabelece que a empresa deve identificar, reunir e analisar dados para demonstrar que o sistema de gestão da qualidade é adequado. Os referidos dados coletados podem servir de base para os projetos Seis Sigma;

- a redução de custos ou a diminuição da variabilidade dos processos de uma determinada organização, ocasionados pela adoção do Seis Sigma, indica a melhoria contínua do sistema de gestão de qualidade, solicitado pela ISO 9001:2000;

- os resultados finais dos projetos Seis Sigma podem ser utilizados como entrada de dados para a análise crítica do sistema de gestão de qualidade pela direção, demonstrando seu interesse e envolvimento, conforme solicitado na norma ISO 9001:2000;

- a principal pretensão do Seis Sigma é aumentar a satisfação dos clientes, utilizando para tanto os projetos que trabalham para a eliminação das Não Conformidades relacionadas com as características críticas para a qualidade de produtos e serviços. O Sistema de Gestão da Qualidade (SGQ) de uma organização, de acordo com as normas ISO 9001:2000, deve estar comprometido permanentemente com o aumento da satisfação dos clientes, característica essa que é totalmente compatível e solicitada na filosofia do programa Seis Sigma;

- o objetivo dos projetos Seis Sigma é elaborar Ações Corretivas e Preventivas, ocasionando melhorias para o SGQ, devidamente verificadas por técnicas estatísticas e por pessoal devidamente especializado, estando compatível com o comprometimento que a direção da organização deve demonstrar com o SGQ e com a melhoria contínua de produtos e serviços. 
As ferramentas de qualidade adotadas nos programas ISO, 9000, ISO 14000 e TQM estão descritas na Tabela 2.

Tabela 2 - Ferramentas adotadas nos programas de qualidade

\begin{tabular}{|c|c|c|c|c|c|c|c|c|c|c|c|c|c|c|c|c|c|}
\hline 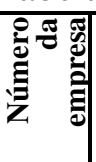 & 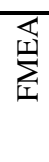 & & 可 & 宅 & 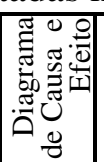 & 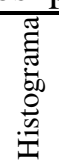 & 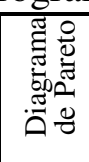 & 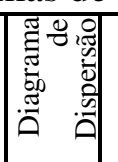 & 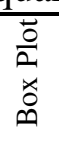 & 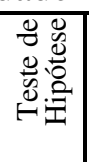 & 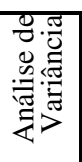 & 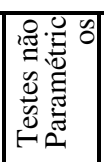 & 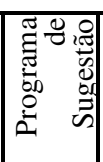 & $\tilde{n}$ & 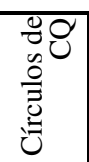 & $\begin{array}{l}\mathbb{U} \\
\text { Q }\end{array}$ & 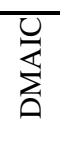 \\
\hline $1-\mathrm{N}$ & & & $\bar{X}$ & & X & 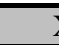 & $\bar{X}$ & & & & & & X & & & & \\
\hline $2-\mathrm{N}$ & & & $\bar{X}$ & & $x$ & $\overline{2}$ & $\bar{X}$ & $\bar{x}$ & & & & & $\bar{x}$ & $\underline{I}$ & & & \\
\hline 3-M & & & $\bar{X}$ & 2 & $\sqrt{x}$ & $\overline{2}$ & $\bar{X}$ & $\bar{x}$ & $\bar{y}$ & $\vec{x}$ & $\bar{X}$ & X & $\sqrt{x}$ & $\bar{\lambda}$ & $\bar{x}$ & & \\
\hline $4 \mathrm{M}$ & & & $\bar{X}$ & & $x$ & $\overline{2}$ & $\bar{X}$ & $\bar{X}$ & & & & & 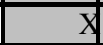 & $\bar{\lambda}$ & & $\bar{x}$ & \\
\hline $5-\mathrm{N}$ & & & $\bar{X}$ & & X & $\bar{x}$ & & & & & & & X & & $\bar{X}$ & $\bar{\lambda}$ & \\
\hline $6-\mathrm{M}$ & & & $\bar{X}$ & & X & $\bar{x}$ & $\bar{z}$ & & & & & & 7 & & & $\bar{x}$ & \\
\hline $7-\mathrm{M}$ & & & $\bar{X}$ & & X & $\overline{2}$ & $\bar{X}$ & $\bar{X}$ & $y$ & X & $\overline{\mathrm{X}}$ & $x$ & X & $\bar{\lambda}$ & $\bar{x}$ & $\bar{y}$ & \\
\hline $8 \mathrm{M}$ & & & $\bar{X}$ & $\overline{2}$ & X & $\overline{2}$ & $\bar{X}$ & $x$ & $\bar{x}$ & & & & X & $\bar{X}$ & & & \\
\hline $9-1$ & & & $\bar{X}$ & & $x$ & $\overline{2}$ & $\bar{X}$ & 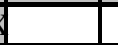 & & & & & X & $\bar{\lambda}$ & & $\bar{y}$ & \\
\hline $10-\mathrm{M}$ & & & $\bar{X}$ & & $\bar{X}$ & $\bar{x}$ & $\bar{X}$ & $\bar{x}$ & & X & $\bar{x}$ & & X & $\bar{\lambda}$ & $\bar{x}$ & $\bar{X}$ & \\
\hline
\end{tabular}

Fonte: as autoras

Analisando as ferramentas adotadas para os diversos programas de qualidade da Tabela 2, observa-se que as empresas nacionais (empresas números 1,2, 5 e 9) utilizam uma quantidade menor de ferramentas de qualidade quando comparadas com as organizações multinacionais (empresas números 3, 4, 6, 7, 8 e 10). Nas empresas nacionais a média é de aproximadamente 8 ferramentas implantadas enquanto que nas organizações multinacionais a média é de 12. Pode-se verificar também que as ferramentas adotadas nas organizações multinacionais são as de maior complexidade (exemplo: QFD-Desdobramento da Função Qualidade e DOE-Delineamento de Experimentos) quando comparadas com as empresas nacionais.

Cabe ressaltar que quatro empresas disseram utilizar a ferramenta DMAIC, apesar de não possuírem o Seis Sigma implantado. A justificativa alegada pelas empresas é que o mesmo é uma boa ferramenta e que pode ser aplicada para outros programas como as normas ISO 9001:2000, a ISO 14001, dentre outros programas. 
As ferramentas de qualidade adotadas no programa Seis Sigma estão descritas na Tabela 3.

Tabela 3 - Ferramentas empregadas no Seis Sigma

\begin{tabular}{|c|c|c|c|c|c|c|c|c|c|c|c|c|c|c|c|c|c|c|}
\hline 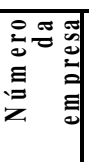 & $\sum_{i=}^{凶}$ & 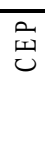 & 売 & 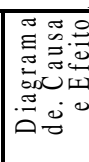 & 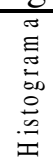 & 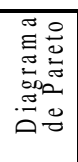 & 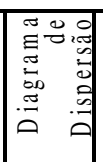 & 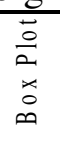 & $\begin{array}{l}0.0 \\
0.00 \\
0.0 \\
0.0 \\
0.0\end{array}$ & 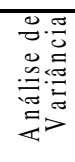 & 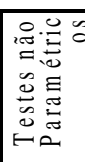 & 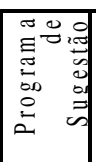 & in & 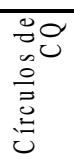 & $\begin{array}{l}\mathbb{4} \\
\tilde{U} \\
0\end{array}$ & 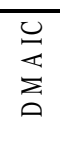 & $\begin{array}{l}n \\
n \\
\omega \\
0\end{array}$ & एٕل \\
\hline$\overline{1-N}$ & & & & & & & $\bar{X}$ & $X$ & $\bar{X}$ & $\bar{X}$ & & & & & & $\bar{X}$ & & \\
\hline $2-\mathrm{N}$ & $\bar{X}$ & $\bar{X}$ & & $\bar{X}$ & $\bar{X}$ & $\bar{X}$ & $\bar{X}$ & $\bar{X}$ & $\bar{X}$ & $\bar{X}$ & $\bar{X}$ & & & & & $\bar{X}$ & & $\bar{X}$ \\
\hline 3-M & $\bar{X}$ & $\bar{X}$ & $X$ & $\bar{X}$ & 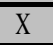 & $\bar{X}$ & $\bar{X}$ & $\bar{X}$ & $\bar{X}$ & $\bar{X}$ & $\bar{X}$ & $\bar{X}$ & $\bar{X}$ & $\bar{X}$ & $\bar{X}$ & $\bar{X}$ & $\bar{X}$ & $\bar{X}$ \\
\hline 4-M & $\bar{X}$ & $\bar{X}$ & & $\bar{X}$ & 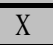 & $\bar{X}$ & $\bar{X}$ & & $\bar{X}$ & $\bar{X}$ & & $\bar{X}$ & $\bar{X}$ & $\bar{X}$ & $\bar{X}$ & $\bar{X}$ & & $\bar{X}$ \\
\hline $5-\mathrm{N}$ & & & & & & & & & $\bar{X}$ & $\bar{X}$ & $X$ & $\bar{X}$ & & $\bar{X}$ & $\bar{X}$ & $\bar{X}$ & & \\
\hline $6-\mathrm{M}$ & $\bar{X}$ & $\bar{X}$ & $\bar{X}$ & $\bar{X}$ & $\bar{X}$ & $\bar{X}$ & $\bar{X}$ & $\bar{X}$ & $\bar{X}$ & $\bar{X}$ & & & & & $\bar{X}$ & $\bar{X}$ & & \\
\hline $7-\mathrm{M}$ & $X$ & $X$ & $X$ & $X$ & $X$ & $X$ & $X$ & $X$ & $X$ & $X$ & $X$ & $X$ & $X$ & $X$ & $X$ & $X$ & $X$ & $X$ \\
\hline 8-M & $X$ & & & $X$ & $X$ & $\bar{X}$ & $X$ & $X$ & $\bar{X}$ & $X$ & & & 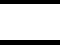 & & $X$ & $X$ & $X$ & $X$ \\
\hline 9-N & $X$ & $X$ & $X$ & $\bar{X}$ & $X$ & $X$ & $\bar{X}$ & $X$ & $\bar{X}$ & $\bar{X}$ & & & & & & $\bar{X}$ & & \\
\hline 10-M & $X$ & $\bar{X}$ & $X$ & $X$ & 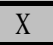 & $X$ & $\bar{X}$ & & $X$ & $\bar{X}$ & & $\bar{X}$ & $\bar{X}$ & $\bar{X}$ & $\bar{X}$ & $\bar{X}$ & & \\
\hline
\end{tabular}

Fonte: as autoras

Ao analisar as ferramentas adotadas para o programa Seis Sigma, na Tabela 3, verifica-se que:

As empresas nacionais (números 1, 2, 5 e 9) utilizam uma quantidade menor de ferramentas de qualidade quando comparadas com as organizações multinacionais (números 3, 4, 6, 7, 8 e 10). Nas empresas nacionais a média é de aproximadamente 9 ferramentas implantadas, enquanto que nas companhias multinacionais a média é de 15 . Pode-se verificar também que as ferramentas adotadas nas organizações multinacionais são as de maior complexidade (exemplo: DOE, DFSS e QFD). O DFSS (Design for Six Sigma) é pouco utilizado nas empresas analisadas com apenas três organizações fazendo sua aplicação.

Cabe ressaltar, que apenas uma das empresas pesquisadas se candidatou uma única vez ao Prêmio Nacional da Qualidade - PNQ, porém não foi vencedora do mesmo.

\section{Verificação da validade das proposições}

Através do estudo de múltiplos casos pode-se avaliar as proposições sugeridos inicialmente neste trabalho, conforme constatação a seguir:

Proposição 1 - O conjunto de ferramentas básicas da qualidade, utilizado nas organizações pesquisadas é o mesmo no programa Seis Sigma e demais programas.

A proposição $P_{1}$ não é verdadeira, pois as empresas que participaram da análise qualitativa demonstraram utilizar diferentes ferramentas da qualidade, conforme descrito na Tabela 4 : 
Tabela 4 - Grau de utilização das ferramentas da qualidade

\begin{tabular}{l|c|c}
\multicolumn{1}{c|}{ Ferramentas da qualidade } & Seis Sigma & $\begin{array}{c}\text { Demais } \\
\text { Programas }\end{array}$ \\
\hline Teste de Hipóteses & Alta & Baixa \\
\hline Análise de Variância & Alta & Baixa \\
\hline DMAIC (Definir, Medir, Analisar, Melhorar e Controlar) & Alta & Não utilizada \\
\hline FMEA (Análise do Modo e Efeito da Falha) & Média & Alta \\
\hline CEP (Controle Estatístico do Processo) & Média & Alta \\
\hline Diagrama de Causa-Efeito (Espinha de Peixe) & Média & Alta \\
\hline Histograma & Média & Alta \\
\hline Diagrama de Pareto & Média & Alta \\
\hline Box Plot & Média & Baixa \\
\hline PDCA (Planejamento, Execução, Controle e Análise) & Média & Alta \\
\hline DFSS (Design for Six Sigma) & Baixa & Não utilizada \\
\hline DOE - Delineamento de Experimentos & Baixa & Baixa \\
\hline QFD (Desdobramento da Função Qualidade) & Baixa & Baixa \\
\hline Programas de Sugestão & Baixa & Alta \\
\hline Círculos de Controle de Qualidade & Baixa & Não utilizada \\
\hline Fona as audasa
\end{tabular}

Fonte: as autoras

Analisando-se a Tabela 4 (a coluna do programa Seis Sigma), verifica-se que as ferramentas da qualidade como Teste de Hipóteses, Análise de Variância e o DMAIC (Define, Measure, Analyze, Design and Verify) são utilizados por todas as empresas que implantaram o Seis Sigma. As ferramentas como FMEA (Análise do Modo e Efeito da Falha), CEP (Controle Estatístico do Processo), Diagrama de Causa-Efeito, Histograma, Diagrama de Pareto, Box Plot e PDCA (Plan, Do, Check and Act) são as ferramentas mais utilizadas nas empresas analisadas. As ferramentas da qualidade como DFSS (Design for Six Sigma), DOE (Delineamento de Experimentos), QFD (Quality Function Deployment), Programas de Sugestão e Círculos de Controle de Qualidade são as ferramentas menos utilizadas.

Avaliando-se a Tabela 4 (a coluna "Demais Programas") (ISO 9001:2000, ISO 14000, TQM, dentre outros), observa-ve que as ferramentas FMEA (Análise do Modo e Efeito da Falha), CEP (Controle Estatístico do Processo), Diagrama de Causa-Efeito, Histograma, Diagrama de Pareto, PDCA (Plan, Do, Check and Act) e Programas de Sugestão são as utilizadas por todas as empresas da análise qualitativa. 


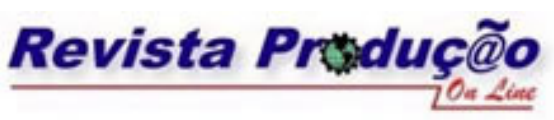

Associação Brasileira de Engenharia de Produção - ABEPRO

Universidade Federal de Santa Catarina - UFSC

www.producaoonline.org.br

ISSN 1676 - 1901 / Vol. IX/ Num.I/2009

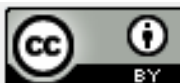

As ferramentas: Teste de Hipóteses, Análise de Variância, Box Plot, DOE (Delineamento de Experimentos) e QFD (Quality Function Deployment) são pouco utilizadas nas empresas estudadas. As ferramentas DMAIC (Define, Measure, Analyze, Design and Verify), DFSS (Design for Six Sigma) e Círculos de Controle de Qualidade não foram implantadas, sendo que as duas primeiras geralmente são específicas do programa Seis Sigma. Deve-se salientar que os programas de qualidade verificados são distintos entre si, porém existe semelhança no grupo de ferramentas da qualidade ou técnicas estatísticas adotados pelos mesmos. Cabe recordar também que as organizações foram escolhidas pelo fato de possuírem histórico de implantação de programas de qualidade, preferencialmente as normas ISO 9001:2000 e o Seis Sigma, implantados há pelo menos dois anos.

Proposição 2 - Existe sinergia entre o Seis Sigma e os demais programas como a ISO 9001:2000, quando utilizados simultaneamente nas empresas.

A proposição $P_{2}$ pode ser considerada como verdadeira, pois as empresas que participaram da análise qualitativa afirmaram existir uma coexistência harmoniosa e o compartilhamento de diversos recursos entre os programas de qualidade adotados.

As sinergias verificadas entre ISO 9000 e o Seis Sigma foram: o Seis Sigma é uma excelente ferramenta para uma melhoria contínua do Sistema de Gestão da Qualidade, exigido pela norma ISO 9001:2000; as auditorias internas do Sistema de Gestão da Qualidade (item obrigatório da norma ISO 9001:2000) podem auxiliar na análise e quantificação dos projetos Seis Sigma, aumentando a credibilidade dos mesmos e os resultados dos projetos Seis Sigma podem ser as bases para a Análise Crítica pela direção da organização.

As sinergias verificadas entre ISO 9000, o Seis Sigma e o TQM foram: o objetivo dos programas ISO 9000, Seis Sigma e TQM é aumentar a satisfação dos clientes externos da empresa; o compartilhamento da documentação do Sistema da Qualidade (Instruções de Trabalho, Procedimentos Operacionais, Especificações de Produtos, dentre outros) e da sistemática de elaboração e controle dos mesmos e o uso comum de diversos equipamentos e de pessoal devidamente qualificado.

Proposição 3 - A implantação do Seis Sigma acarretou benefícios financeiros significativos para as empresas que o adotou. 
As empresas pesquisadas, na sua totalidade, indicaram que obtiveram benefícios financeiros com a implantação do Seis Sigma, já nos primeiros anos de sua implantação, sendo que o valor total investido foi inferior aos ganhos proporcionados com o referido programa. Para a validação dos ganhos financeiros oriundos dos projetos Seis Sigma, todas as organizações utilizaram o departamento de contabilidade da empresa, sendo que a periodicidade da validação dos projetos teve grande oscilação conforme demonstra a Tabela 5.

Analisando a Tabela 6, pode-se verificar que as empresas que adotaram o Seis Sigma à mais de 36 meses possuem retorno financeiro mais acentuado, pois os gastos iniciais com treinamentos básicos, compra de programas de computador, dentre outras despesas já foram amortizados. Cabe salientar que o caso número 2, demonstrado na Tabela 5, está calculando o retorno financeiro na forma de redução nos custos da Não-qualidade $(0,5 \%$ no caso), sendo que essa redução é maior que os valores financeiros investidos. 


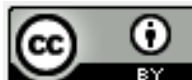

Tabela 5 - Valores investidos, retorno financeiro, templo de implantação e periodicidade de validação dos projetos Seis Sigma dos estudos de caso.

\begin{tabular}{|c|c|c|c|c|}
\hline Caso & $\begin{array}{c}\text { Valores Investidos } \\
\text { (reais) }\end{array}$ & $\begin{array}{c}\text { Retorno Financeiro } \\
\text { (reais) }\end{array}$ & $\begin{array}{c}\text { Tempo de } \\
\text { implementação do } \\
\text { programa Seis } \\
\text { Sigma (meses) }\end{array}$ & $\begin{array}{l}\text { Periodicidade } \\
\text { de validação } \\
\text { dos projetos } \\
\text { (meses) }\end{array}$ \\
\hline 1 & Não informado & $\begin{array}{l}\text { Não informado, } \\
\text { porém é superior ao } \\
\text { valor investido }\end{array}$ & 26 & 12 \\
\hline 2 & $\begin{array}{c}\text { Entre } 250.000 \mathrm{a} \\
499.000\end{array}$ & $\begin{array}{c}\text { Redução de } 0,5 \% \text { nos } \\
\text { custos da Não- } \\
\text { Qualidade }\end{array}$ & 24 & 3 \\
\hline 3 & $\begin{array}{c}\text { Entre } 100.000 \mathrm{a} \\
249.000\end{array}$ & 17 Milhões por ano & 25 & 6 \\
\hline 4 & $\begin{array}{c}\text { Entre } 100.000 \text { a } \\
249.000\end{array}$ & Acima de 5 Milhões & 72 & 6 \\
\hline 5 & $\begin{array}{c}\text { Entre } 100.000 \mathrm{a} \\
249.000\end{array}$ & Acima de 500.000 & 26 & 3 \\
\hline 6 & $\begin{array}{l}\text { Entre } 100.000 \mathrm{a} \\
249.000\end{array}$ & Acima de 500.000 & 24 & 12 \\
\hline 7 & Mais de 1 Milhão & Mais de 5 Milhões & 36 & 24 \\
\hline 8 & $\begin{array}{c}\text { Entre } 100.000 \text { a } \\
249.000\end{array}$ & Acima de 500.000 & 60 & 36 \\
\hline 9 & $\begin{array}{c}\text { Entre } 500.000 \mathrm{a} \\
\mathrm{R} \$ 1.000 .000\end{array}$ & Mais de 5 Milhões & 36 & 01 \\
\hline 10 & $\begin{array}{c}\text { Entre } 250.000 \text { a } \\
499.000\end{array}$ & $\begin{array}{c}\text { Superior ao } \\
\text { investido, porém não } \\
\text { foi informada a } \\
\text { quantia aproximada }\end{array}$ & 48 & 01 \\
\hline
\end{tabular}

\section{CONSIDERAÇÕES FINAIS}

A pesquisa qualitativa indicou os principais aspectos da adoção do Seis Sigma em 10 (dez) grandes empresas brasileiras, indicando que as mesmas utilizam a nomenclatura específica da metodologia (black belts, green belts, dentre outros); adotam as ferramentas da qualidade como DMAIC, análise de variância, teste de hipótese, diagrama de dispersão, dentre outras, foi verificado também que as organizações que implantaram o Seis Sigma à mais de quatro anos, adotaram ferramentas da qualidade de maior complexidade; as áreas contábeis realizam 
periodicamente o controle financeiro dos projetos; houve uma vasta divulgação interna do programa; realizam um amplo treinamento para todos os envolvidos; existe um grande apoio por parte da direção das empresas ao Seis Sigma; e existe a perspectiva de ampliação do programa para outras áreas.

A primeira proposição "o conjunto de ferramentas básicas da qualidade, utilizado nas organizações pesquisadas é o mesmo para o programa Seis Sigma e demais programas", foi considerado como não verdadeiro, pois as empresas demonstraram utilizar diferentes ferramentas da qualidade, porém técnicas consagradas como FMEA; CEP; Diagrama de Causa-Efeito; Histograma; Diagrama de Pareto; PDCA e Programas de Sugestão são amplamente empregadas em todas as empresas avaliadas, indicando que as empresas do estudo de caso optaram por utilizar as ferramentas da qualidade mais simples, mesmas as organizações que implantaram o Seis Sigma à mais de 24 meses.

A segunda proposição "existe sinergia entre o Seis Sigma e os demais programas como a ISO 9001:2000, quando utilizados simultaneamente nas empresas", foi considerado como verdadeiro, pois as organizações afirmaram possuir diversos aspectos em comum, compartilhando recursos materiais e pessoais, dados e informações. Os aspectos mais relevantes que sugerem sinergia entre os programas de qualidade são: o Seis Sigma é uma excelente ferramenta para a melhoria contínua do Sistema de Gestão da Qualidade (SGQ), exigido pela norma ISO 9001:2000; as auditorias internas do SGQ podem auxiliar na análise e na quantificação dos projetos Seis Sigma, aumentando a credibilidade dos mesmos e os resultados dos projetos Seis Sigma podem ser as bases para a Análise Crítica pela direção da organização (item exigido pela norma ISO 9001:2000).

A terceira proposição "a implantação do Seis Sigma acarretou benefícios financeiros significativos para as empresas que o adotou", foi considerado como verídico, pois as análises qualitativas demonstraram que a implantação do Seis Sigma nas dez grandes empresas estudadas empregou na adoção do referido programa vastos recursos financeiros (na compra ou desenvolvimento de programas estatísticos, em diversos tipos de treinamentos, dentre outros) e humanos (pessoal com diversas categorias de dedicação de tempo ao programa, colaboradores recebendo considerável quantidade de treinamentos específicos), porém em todas as organizações 
houve o consenso de que os resultados proporcionados pelo programa foram maiores que os valores investidos.

Nas empresas visitadas houve consenso na afirmação de que o Seis Sigma contribuiu significativamente para a melhoria da cultura organizacional, pois ocasionou melhorias visíveis de qualidade, produtividade e ganhos financeiros relevantes.

A avaliação aplicada neste estudo apresenta as limitações inerentes ao método de pesquisa adotado, ou seja, os resultados obtidos não devem ser generalizados, porém acredita-se que os mesmos possam contribuir significativamente para um maior e melhor entendimento dos fatores que exercem influências nos programas de melhoria da qualidade adotados nas empresas brasileiras.

A atual pesquisa abordou dez grandes empresas brasileiras, portanto, poderia ser confeccionado um trabalho abrangendo as pequenas e médias organizações, visando uma comparação. Outro futuro projeto seria a aplicação da atual metodologia em outros países, objetivando a comparação entre nações.

\section{AGRADECIMENTOS}

As autoras agradecem ao CNPq e à FAPESP pelo financiamento parcial desta pesquisa.

\section{REFERÊNCIAS}

BASU, R. Six-Sigma to operational excellence: role of tools and techniques. International Journal of Six Sigma and Competitive Advantage, v.1, n.1, p.44-64, 2004.

BEHESHTI, H. M., LOLLAR, J. G. An empirical study of US SMEs using TQM. Total Quality Management \& Business Excellence, v.14, n.8, p.839-847, Oct. 2003.

BLAKESLEE JR, J. A. Achieving quantum leaps in quality and competitiveness: implementing the Six Sigma solution in your company. In: ASQ'S ANNUAL QUALITY CONGRESS PROCEEDINGS, 53., 2001, USA. Anais. USA: ASQ's, 2001.

CARVALHO, M. M.; PALADINI, E. P. Gestão da qualidade: teoria e casos. Rio de Janeiro: Editora Campus, 2005. 
CHOW-CHUA, C., GOH, M., WAN, T. B. Does ISO 9000 certification improve business performance? International Journal of Quality \& Reability Management, v.20, n.8, p.936953, Nov. 2003.

COOPER, R. D.; SCHINDLER, P. S. Método de pesquisa em administração. ed. 7. Porto Alegre: Bookman, 2003.

ECKES, G. A revolução Seis Sigma. Rio de Janeiro: Campus, 2001.

HENDRICKS, K. B.; Singhall, V. R. Does implementing an effective TQM program actually improve operating performance? - Empirical evidence from firms that have won quality awards. Management Science, v.43, n.9, p.258-1274, 1997.

HENDRICKS, K. B.; SINGHALL, V. R. The long-run stock price performance of firms with effective TQM programs. Management Science, v.47, n.3, p.359-369, 2001.

HOERL, R., SNEE, R. D. Statistical thinking: improving business performance. Duxbury Press, Pacific Grove, CA: 2002.

GARVIN, D. A. Managing quality: the strategic and competitive edge. EUA, New York: Harvard Business School, 1988. 319 p.

GUILLÉN, M. F. The age of ecletism: current organization trends and the evolution of management models. Sloan Management Review, v.36, n.1, p.75-86, Fall 1994.

LEE, Y., LAZARUS, H. Uses and criticisms of total quality management. Journal of Management Development, v.12, n.7, p.5-10, 1996.

LI-HSING, H., CHEN-CHIA, C. A study of implementing Six Sigma quality management system in government agencies for raising service quality. Journal of American Academy of Business, v.10, n.1, p.167-173, Sep. 2006.

LINDERMAN, K., SCHROEDER, R. G., CHOO, A. S. Six Sigma: the role of goals in improvement teams. Journal of Operations Management, v. 24, n. 6, p. 779-790, Dec. 2006.

KAYNAK, H. The relationship between total quality management practices and their effects on firm performance. Journal of Operations Management, v. 21, n. 4, p. 405-435, July 2003.

MONTES, F. J. L., JOVER, A. V., FERNÁNDEZ, L. M. M. Factors affecting the relationship between total quality management and organizational performance. International Journal of Quality \& Reability Management, v.20, n.2, p.188-208, Nov. 2002.

PANDE, P. S.; NEUMAN R. P.; CAVANAGH R. R. Six Sigma way team field book, the an implementation guide for project improvement. New York: McGraw-Hill, 2001. 
PFEIFER, T.; RESSIGER, W.; CANALES, C. Integrating Six Sigma with quality management systems. The TQM Magazine, v.16, n.4, p.241-249, 2004.

POWER, T. C. Total quality management as competitive advantage: a review and empirical study. Strategic Management Journal, v.16, n.1, p.15-37, 1997.

PRAHALAD, C. K; HAMEL, G. The core competence of the corporation. Harvard Business Review, p. 79-91, May/June 1990.

PRAJOGO, D. I., SOHAL, A. S. The sustainability and evolution of quality improvement programmers - an Australian case study. Total Quality Management, v.15, n.2, p.205-220, Mar. 2004.

RAJAN, M., TAMIMI, N. Payoff to ISO 9000 registration. Journal of Investing. p.71-78, Spring 2003.

RAVICHANDRAN, J. Six Sigma milestone: an overall sigma level of an organization. Total Quality Management \& Business Excellence, v.17, n.8, p.973-980, Oct. 2006.

REED, R.; LEMAK, D. J.; MERO, N. P. Total quality management and sustainable competitive advantage. Journal of Quality Management, n. 5, p. 5-26, 2000.

SNEE, R. D. Six-sigma: the evolution of 100 years of business improvement methodology. International Journal of Six Sigma and Competitive Advantage, v.1, n.1, p.4-20, 2004.

SPENCER, B. A. Models of organization and total quality management: a comparison and critical evolution. Academy of Management Review, v.19, n.3, p.446-471, July, 1996.

WILSON, P. J., WALSH, M. A. T., MEEDY, K. S. An examination of the economic benefits of ISO 9000 and the Baldrige award to manufacturing firms. Engineering Management Journal, p.03-11, Dec. 2003.

YIN, R. K. Estudo de caso - planejamento e métodos. São Paulo: Bookman, 2004. 